\title{
The SMAs: neural substrate of the temporal accumulator?
}

\section{Laurence Casini and Franck Vidal* \\ Laboratoire de Neurobiologie de la Cognition, Aix-Marseille Université, CNRS, Marseille, France \\ *Correspondence: franck.vidall@univ-provence.fr}

One of the most widely cited models in time estimation is the "pacemaker-counter clock" which consists of a pacemaker generating pulses and an accumulator in which pulses are stored. The level reached in this accumulator at the end of an interval to be estimated sets the subjective elapsed time. Although this model is able to accurately describe temporal performance, it can nevertheless be considered no more than a good metaphor of actual temporal processing mechanisms. Finding specific brain areas behaving as a pacemaker and/or as an accumulator would provide additional support for this model. Evidence has now been amassed suggesting that the SMAs could be a neural substrate of the accumulator.

\section{EEG EVIDENCE FOR A CUMULATIVE PROCESS IN THE SMA}

In two different timing tasks (a production and a discrimination one) Macar et al. (1999) showed that the mesial frontal cortex, including the SMAs, behaves in an accumulator-like way. The authors recorded the contingent negative variation (CNV: Walter et al., 1964) an electrical activity of the brain which develops between two events of interest separated by a predictable time interval. In line with the results of Casini and Macar (1997), the authors hypothesized that spontaneous fluctuations of brain activation should influence the speed of the pacemaker, determining the number of accumulated pulses and, hence, subjective temporal estimation. Using the $\mathrm{CNV}$ as an index of the accumulation process, Macar et al. (1999) observed that the larger the estimated duration, the larger the CNV over the SMAs (Figure 1). These observations, suggesting that the SMAs act as an accumulator, have since been reproduced with identical intervals (Macar and Vidal, 2002) and with shorter intervals involving the auditory modality (Bendixen et al., 2005). Moreover, Noguchi and Kakigi (2006) recorded the magnetic counterpart of the CNV in a timing task and observed the same magnetic build-up of activity around the SMAs during the interval to be estimated. In addition, Noguchi and Kakigi found a positive correlation between the amplitude of the visual evoked magnetic field (VEF) and the size of the CNV-like field. If one hypothesizes that the amplitude of the VEF can be interpreted as an index of activation, a larger VEF is representative of a larger activation which, in agreement with the concept proposed by Macar et al. (1999) should be associated with more accumulated pulses and, hence, a larger magnetic CNV.

\section{FMRI EVIDENCE FOR A CUMULATIVE PROCESS IN THE SMA}

Functional magnetic resonance imaging data also point toward the SMAs playing a key role in time processing. According to Zakay (1989), the more attention is paid to time, the more pulses are accumulated, which accounts for the well known effects of attention on timing performance (e.g., Casini and Macar, 1996; for a review see Brown, 1997). Coull et al. (2004) showed that when the amount of attention is explicitly controlled SMA activity positively correlates with attention paid to timing. SMAs therefore behave as the accumulator postulated by the model. In addition, in reproduction tasks where time has to be successively coded and retrieved, the pacemaker-counter model postulates that the accumulator is necessarily switched on at the beginning of both stages (coding and retrieval). Several brain areas have now been shown to be active in a reproduction task. However, the SMAs were the only area to be engaged during both stages of the task (Figure 2; Coull et al., 2008). As such, the SMAs seem to be the only structure where the accumulation process can take place.

\section{SINGLE CELL RECORDINGS EVIDENCE FOR A CUMULATIVE PROCESS IN THE SMA}

Further evidence supporting the presence of a cumulative process in SMAs used for timing can be found in single unit recordings of awake monkeys, both in temporal production and estimation. In a production task, Mita et al. (2009) found neurons in the SMA and pre-SMA exhibiting decay

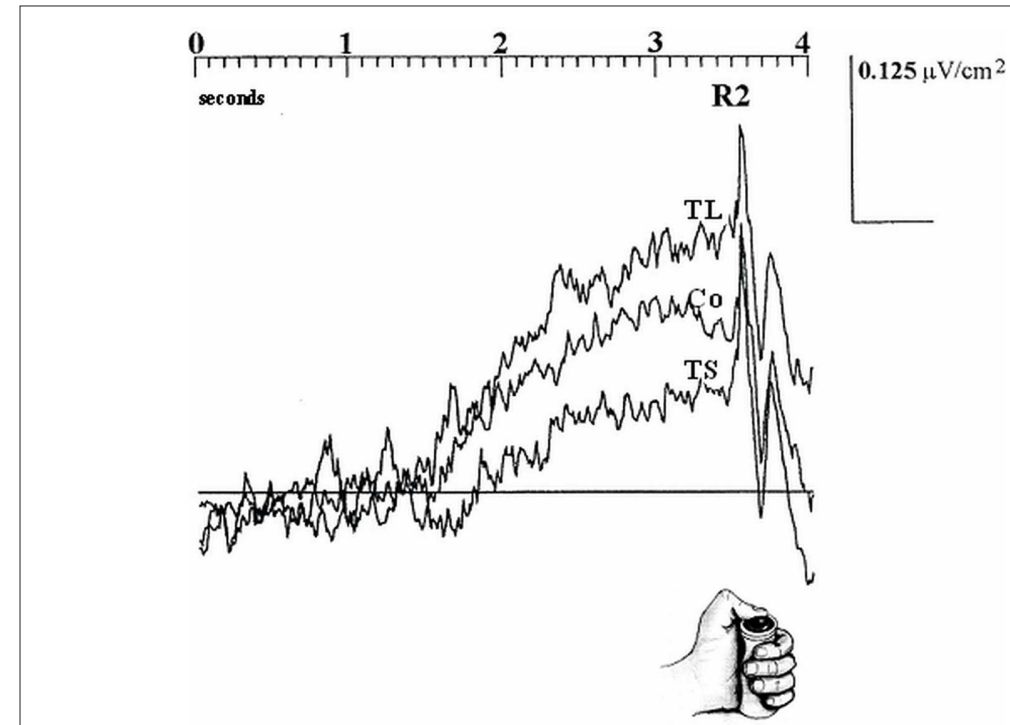

FIGURE 1 | Electrophysiological activity over the SMAs in 10 subjects during a time production task as a function of performance (TL, "too long"; Co, "correct";TS, "too short") time-locked to the end (button press) of the interval. Amplitude (negative up) on ordinates, time on abscissas. Adapted from Macar et al., 1999. 
or build-up patterns evoking a cumulative process. In these so called "time graded" neurons the longer the interval to be produced, the higher the firing rate at the end of the interval (Figure 3B). In addition, Akkal et al. (2004) recorded neurons in the pre-SMA during a reaction time task using fixed or variable foreperiods. Only when

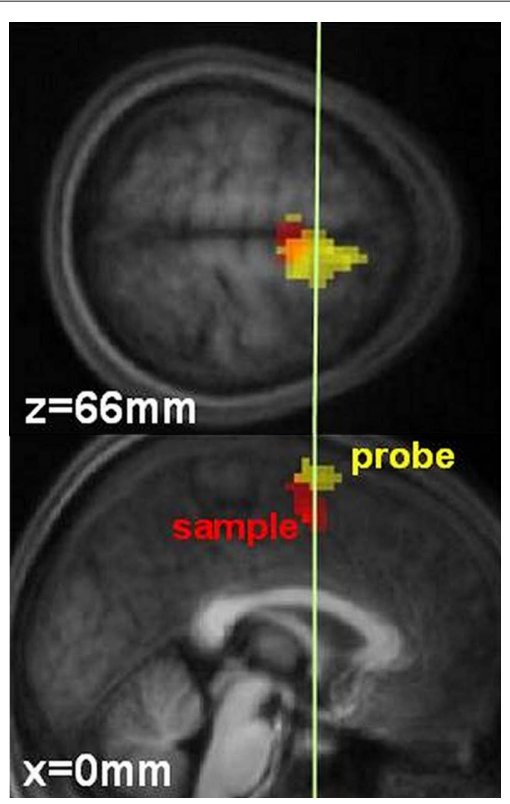

FIGURE 2 |Temporal processing by the SMAs in 14 subjects. Red represents activation obtained during sample stimuli presentation. Yellow represents activation obtained during probe stimuli presentation. The white line $(y=0 \mathrm{~mm})$ denotes the border between the SMA proper and the pre-SMA. X and Z correspond to the coordinates of each slice. Adapted from Coull et al. (2008).
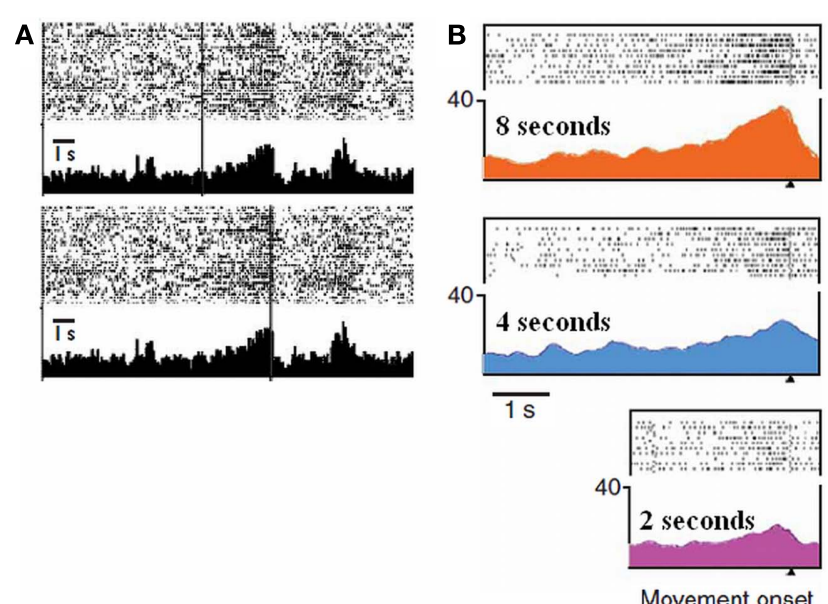

FIGURE 3 | Single unit activities as a function of elapsed time in a time estimation task (A) (adapted from Akkal et al., 2004) and in a production task (B) (adapted from Mita et al., 2009). (A) Raster displays and peri-event histograms: The figure represents an example of pre-SMA cells showing build-up neuronal activity as time elapses. The vertical bars indicate the onset (top) and the end (bottom) of the interval. Note that this pattern only occurs when the end of the interval can be predicted, that is when this interval is fixed. (B) Raster displays and spike-density functions: The figure represents an example of pre-SMA cells showing graded neuronal activity that depended on the time intervals to be produced: highest during a 8-s interval, moderate during a 4-s interval, and lowest during a 2-s interval. The displays are aligned according to the end of the produced interval. the foreperiod was fixed, i.e., only when foreperiod duration could be accurately predicted, did pre-SMA neurons behave in an accumulator-like way: During the fixed foreperiod, neuronal activity exhibited a particular build-up pattern culminating at the end of the delay (Figure 3A). The authors concluded that the build-up pattern they observed could “... represent the neuronal substrate of the temporal accumulator property proposed by previous authors" (page, 1286). Indeed, the neuronal patterns observed in Figure 3 are reminiscent of the time course of the $\mathrm{CNV}$ displayed in Figure 1.

\section{OTHER TIMING PROCESSES OUTSIDE THE SMAS?}

Where could pulses stored in the SMAs come from? Several arguments provided by animal as well as patient studies indicate that the basal ganglia could host the pacemaker system (for reviews see Buhusi and Meck, 2005; Coull et al., 2011). For example, dopaminergic drugs are known to selectively affect the speed of an internal clock in both animals and humans. Moreover, patients with Parkinson's disease show impaired temporal performance in both motor and sensory timing within both the second and sub-second time ranges. It should be noted that the SMAs are one of the main targets of the basal ganglia via the thalamus.

Finally, it is often assumed that to provide accurate timing, in addition to specific "clock" processes, distinct non-specific memory, and decisional components are also required. When subjects have to memorize a "target" duration and judge whether a probe interval is shorter, equal or longer, the $\mathrm{CNV}$ recorded during the probe ends at the moment when the target would end, even when the probe lasts longer (Macar and Vidal, 2003; Pfeuty et al., 2003). It is noteworthy that during these longer probes no external information indicates that the target duration is over. Therefore, this effect may only be explained by assuming subjects used internal information which can be viewed as temporal memory. However, Macar and Vidal (2003) showed that this temporal memory effect was not implemented by the SMAs but in other, not currently fully identified, structures. In sum, the functional role of the SMA in timing seems well confined to the cumulative process. 


\section{REFERENCES}

Akkal, D., Escola, L., Bioulac, B., and Burbaud, P. (2004). Time predictability modulates pre-supplementary motor area neuronal activity. Neuroreport 15, 1283-1286.

Bendixen, A., Grimm, S., and Schroger, E. (2005). Human auditory event-related potentials predict duration judgments. Neurosci. Lett. 383, 284-288.

Brown, S. W. (1997). Attentional resources in timing: Interference effects in concurrent temporal and nontemporal working memory tasks. Percept. Psychophys. $59,1118-1140$.

Buhusi, C. V., and Meck, W. H. (2005). What makes us tick? Functional and neural mechanisms of interval timing. Nat. Rev. Neurosci. 6, 755-765.

Casini, L., and Macar, F. (1996). Prefrontal slow potentials in temporal compared to nontemporal tasks. J. Psychophysiol. 10, 252-264.

Casini, L., and Macar, F. (1997).Effect of attention manipulation on judgments of duration and of intensity in the visual modality. Mem. Cognit. 25, 812-819.

Coull, J. T., Cheng, R., and Meck, W. H. (2011). Neuroanatomical and neurochemical substrates of timing. Neuropsychopharmacol. Rev. 36, 3-25.
Coull, J. T., Nazarian, B., and Vidal, F. (2008). Timing, storage and comparison of stimulus duration engage discrete anatomical components of a perceptual timing network. J. Cogn. Neurosci. 20, 2185-2197.

Coull, J. T., Vidal, F., Nazarian, B., and Macar, F. (2004). Functional anatomy of the attentional modulation of time estimation. Science 303, 1506-1508

Macar, F., and Vidal, F. (2002). Time processing reflected by EEG surface Laplacians. Exp. Brain Res. 145, 403-406.

Macar, F., and Vidal, F. (2003). The CNV peak: an index of decision making and temporal memory. Psychophysiology 40, 950-954.

Macar, F., Vidal, F., and Casini, L. (1999). Performancedependent ERP changes in judgments of brief durations. Exp. Brain Res. 125, 271-280.

Mita, A., Mushiake, H., Shima, K., Matsuzaka, Y., and Tanji, J. (2009). Interval time coding by neurons in the presupplementary and supplementary motor areas. Nat. Neurosci. 12, 502-507.

Noguchi, Y., and Kakigi, R. (2006). Time representation can be made from nontemporal information in the brain: an MEG study. Cereb. Cortex 16, 1797-1808.

Pfeuty, M., Ragot, R., and Pouthas, V. (2003). When time is up: $\mathrm{CNV}$ time course differentiates the role of the hemispheres in the discrimination of short tone durations. Exp. Brain Res.151, 372-379.

Walter, W. G., Cooper, R., Aldridge, V. J., McCallum, W. C., and Winter, A. L. (1964). Contingent negative variation: An electrical sign of sensorimotor association and expectancy in the human brain. Nature 203, 380-384.

Zakay, D. (1989). "Subjective and attentional resource allocation: an integrated model of time estimation" in Time and Human Cognition, ed. I. Levin and D. Zakay (North-Holland: Elsevier Science Publishers Eds.), 365-397.

Received: 26 July 2011; accepted: 26 July 2011; published online: 11 August 2011.

Citation: Casini L and Vidal F (2011) The SMAs: neural substrate of the temporal accumulator? Front. Integr. Neurosci. 5:35. doi: 10.3389/fnint.2011.00035

Copyright (c) 2011 Casini and Vidal. This is an open-access article subject to a non-exclusive license between the authors and Frontiers Media SA, which permits use, distribution and reproduction in other forums, provided the original authors and source are credited and other Frontiers conditions are complied with. 